\title{
Inequidades múltiples y persistentes en el campo de la reproducción asistida
}

\section{Multiple and Persistent Inequalities in the Field of Assisted Reproduction}

\author{
Mariana VIERA CHERRO \\ Centro de Estudios Interdisciplinarios Latinoamericanos \\ Facultad de Humanidades y Ciencias de la Educación \\ Universidad de la República (Uruguay) \\ marianaviera@yahoo.com
}

Recibido: 30 de marzo de 2011

Aceptado: 10 de noviembre de 2011

\section{Resumen}

El presente artículo representa una primera reflexión ${ }^{1}$ sobre algunos de los cambios que se están produciendo en los sentidos otorgados a la reproducción, la sexualidad y el parentesco a partir de la existencia y aplicación de las tecnologías de reproducción asistida ${ }^{2}$ en el Uruguay actual. Nos interesa pensar en qué medida estos cambios suponen transformaciones estructurales o transformaciones de superficie que habilitan nuevas desigualdades sobre la geografía de las viejas (Reygadas, 2008).

Palabras clave: reproducción asistida, desigualdades, injusticia, género.

\begin{abstract}
This paper represents a first reflection on some of the changes taking place in the senses given to reproduction, sexuality and relationship from the existence and application of assisted reproductive technologies in the current Uruguay. We want to think about how these changes involve structural changes or changes of surface enable new inequalities on the geography of the old (Reygadas, 2008).
\end{abstract}

${ }^{1}$ Las reflexiones del presente artículo forman parte del proyecto de tesis de maestría —aún en curso-, titulado "Lejos de París. Maternidades, paternidades, derechos reproductivos y tecnologías de reproducción asistida en el Rio de la Plata" que se desarrolla en la Facultad de Humanidades y Ciencias de la Educación de la Universidad de la República (Uruguay).

${ }^{2}$ De aquí en adelante, TRA. Las TRA se definen "como el conjunto de técnicas que, desde el campo interdisciplinario de la medicina terapéutica o de intervención y la medicina experimental, se proponen como una respuesta, más o menos efectiva en términos de sus resultados, a la ausencia de hijos no voluntaria de individuos o parejas" Lucía Ariza (2007:257; en Schwarz, 2009). Suponen intervenciones de baja complejidad como monitoreo y estimulación de la ovulación e inseminación artificial - con vínculo biológico del padre (IA, por sus siglas en inglés) o por donación anónima de esperma (IAD, por sus siglas en inglés) - , hasta otras más complejas: la fecundación in vitro (FIV), la transferencia intratubaria de gametos (TIG o GIFT), la transferencia de embrión a la trompa (TET), la inyección intracitoplasmática de espermatozoide (ICSI) y la transferencia a la trompa de ovocitos microinyectados (TOMI). 
Keywords: assisted reproduction, inequalities, injustice, gender.

Referencia normalizada: Viera Cherro, M. (2012). "Inequidades múltiples y persistentes en el campo de la reproducción asistida”. Revista de Antropología Social, 21, 251-271.

SUMARIO: 1. Inequidades y TRA: advertencias desde el feminismo. 2. El contexto uruguayo. 3. Ovocitos: el cuello de botella de la reproducción asistida. 4. El importunismo de quienes mantienen vínculos "naturalmente infértiles". 5. Los inoportunos. 6. La asistencia para la maternidad como contralor de conductas indeseables. 7. Otras desigualdades: guetización del conocimiento y ponderación de los temas de agenda. 8. Reflexiones para continuar la reflexión. 9. Referencias bibliográficas.

\section{Inequidades y TRA: advertencias desde el feminismo}

Señala Verena Stolcke (2006) que es en el campo del desarrollo biotecnológico donde quizás podamos comenzar a encontrar respuestas acerca de las circunstancias históricas que permiten que las diferencias de sexo generen desigualdades de valor y poder entre los seres humanos. La disputa sobre la manipulación de materiales genéticos aparentemente "desencarnados" evidencia la carga de significados socioculturales que los mismos comportan. ¿Cuánto vale un ovocito? ¿Es posible intercambiarlo? ¿Por qué?

Primero la píldora anticonceptiva habilitó la separación entre el ejercicio de la sexualidad y la reproducción; de manera más contemporánea las tecnologías de reproducción asistida y otras tecnologías como la clonación abrieron una brecha inmensa entre el ejercicio de la sexualidad, la reproducción y el cuerpo. Los seres humanos así engendrados, los "cyborg", cuestionan asimismo las relaciones de parentesco. Estas se entienden, según Butler (2003) como un conjunto de prácticas vinculadas a la reproducción de la vida y las formas de dependencia entre los seres humanos: ¿debería un donante de semen ser responsable por la enfermedad de una criatura nacida con la contribución de su material genético?, es una de las múltiples preguntas que se abren ante este horizonte. Y pensando desde la dimensión de la cultura en tanto que universo de sentido, ¿hasta qué preguntas le permitiremos a la tecnología que nos conduzca?, ¿qué respuestas, como cultura, estamos habilitados/ as a dar?

En los años sesenta y setenta del siglo XX, el feminismo comenzó a interrogarse sobre el lugar del cuerpo y la sexualidad en el análisis de las formas de dominación, cuando el desarrollo de tecnologías penetraba en todos los ámbitos de la vida (Scavone, 2007).

En los años noventa, feministas europeas y estadounidenses comenzarían a criticar algunas de las aristas de desarrollo y aplicación de las TRA (Técnicas de Reproducción Asistida): el sometimiento del cuerpo femenino para lograr una maternidad sin medir costos físicos y psíquicos y resumiendo una vez más a la mujer en su papel de madre; la construcción de un deseo de maternidad al servicio de otro, el

3 “Un cyborg es un organismo cibernético, un híbrido de máquina y organismo, una criatura de realidad social y también de ficción" (Haraway, 1991:149). 
de paternidad, asociado este último a la virilidad; la sobrevaloración del vínculo de sangre por sobre las relaciones de crianza, entre otros aspectos (ibid, 2007)

En la actualidad, otras situaciones habilitan a replantear las relaciones de dominación que las TRA perpetúan o profundizan, ya no solo entre individuos sino entre naciones. Un ejemplo de esto último es el turismo reproductivo en India (Amador Jimenez, 2010). Los bajos costos de las clínicas en ese país y lo poco que se abona a las mujeres subrogantes, sumado a una legislación poco restrictiva, habilita que muchas parejas realicen parte del tratamiento in vitro en India, la parte relativa al alquiler de vientre, mientras que los óvulos, y cuando se necesita también el esperma, proceden de países del denominado Primer Mundo reproduciendo así patrones eugenésicos que valoran determinados rasgos fenotípicos - y se supone también de personalidad - sobre otros que se definen como indeseados (ibid). Estas acciones además reproducen violencias de género, ya que las mujeres subrogantes están a merced del poder de los médicos, de las parejas contratantes y de sus maridos, que en la mayoría de los casos son los que firman el contrato y se encargan de que la mujer cumpla con todos los requisitos para que el embarazo llegue a buen término (ibid). Como contracara las propias mujeres señalan que muchas veces estos embarazos les permiten gozar durante ese tiempo de buena alimentación, vivienda y descanso. Esta práctica replantea asimismo el valor diferente que tiene un feto concebido sin mediar tecnología alguna y que en muchas ocasiones es abortado - la legislación india así lo permite y en general se elige abortar los de sexo femenino-, frente a otro subrogado que debe llegar a término (ibid).

¿Cuáles son las inequidades que se plantean en la aplicación de las TRA en Uruguay? ¿Cuáles son sus particularidades con relación a otros contextos o en qué medida estas prácticas nos permiten, justamente a partir de su especificidad, establecer parangones analizando los sentidos de las mismas en términos de producción de desigualdad? Retomando a Verena Stolcke (2006:543): ¿Qué puede enseñarnos entonces todo el entramado de motivaciones científicas y económicas de significados simbólicos e ilusiones sociales que rodea una biotécnica (...) ¿Y antes todo, cuáles son las consecuencias para las mujeres del hecho de que el cuello de botella de la experimentación biotecnológica es la disponibilidad de ovocitos?

\section{El contexto uruguayo}

En Uruguay no existe una ley que regule la aplicación de las diversas tecnologías de reproducción asistida. En julio de 2003 se presentó una iniciativa que recién se discutió y aprobó en la Cámara de Senadores en $2008^{4}$, no habiendo obtenido luego la aprobación de la Cámara de Diputados.

${ }^{4}$ El Proyecto de Ley fue presentado por el Senador Alberto Cid a la Cámara de Senadores el 17 de octubre de 2008; habían existido iniciativas en el mismo sentido en Uruguay desde 1991 (Proyecto "Reproducción Asistida-Modificación del Código Civil, presentado por los doctores Hugo Batalla y Carlos Cassina), pero sin concretarse un marco legal para el desarrollo de las actividades de las cuatro clínicas que llevan adelante tratamiento de este tipo en Uruguay, además del Banco de Esperma. 
Las prácticas reproductivas en este ámbito se rigen básicamente por los controles que ejerce la propia comunidad médica que trabaja en el área. Uno de los ginecólogos entrevistados, que participó como técnico en la elaboración del mencionado proyecto, reflexionaba sobre la ausencia de controles legales,

Si hasta ahora en 25 años no tuvimos nada y no hubo ningún tipo de problema/y depende un poco del concepto de grupo también, porque cuando vos sos 14 o 15 personas en un grupo ehhh, se presta a que el control y autocontrol de las decisiones, de las posibilidades, se controlen unos con otros, cuando son grupos de $2 \mathrm{o}$ 3 personas no sabés qué puede pasar, entonces por ahí se manejan medicamentos para uno y otros le presta para otros, y después estos óvulos quedaron de acá y se los puedo prestar... estas cosas, en grupos así chicos, no es lo mejor. No digo que funcionen así todos pero el riesgo está y ahí sí es bueno que exista una reglamentación. Yo fui Presidente de la Sociedad de Reproducción, hace muchos años, cuando la creamos, y se creó una forma de solicitud que teníamos para mandar a Salud Pública, un proyecto... yo diría no de ley pero de reglamentación, que estuvieran controlados los centros porque era necesario saber lo qué se hace, cómo se hace y cuáles eran los beneficios que das y qué resultados tenés. Desde el punto de vista de nuestro grupo, nosotros estamos afiliados a una sociedad interamericana que es/hay tres grupos: un europeo, uno latinoamericano y otro interamericano, que nosotros reportamos anualmente todos los casos que tenemos y ahí Uruguay hace muchos años que está representado por el grupo nuestro con la posibilidad de que te hagan autoevaluaciones. O sea, viene gente, que viene un biólogo y un médico y que cada tres años te hace una evaluación de cómo marcha la cosa. Se quedan acá dos días o tres y te dan el aval.

A partir de entrevistas realizadas a médicos y médicas que trabajan en reproducción asistida y algunas mujeres ${ }^{5}$ que se realizaron tratamientos, algunos exitosos y otros no, pudimos definir dos maneras de trabajar en Uruguay en reproducción asistida $^{6}$. Una representada por el médico entrevistado que a su vez formó, a fines de la década de 1980 principios de los años noventa, a otros pocos especialistas, una de las cuales también fue entrevistada y que trabajan los casos de manera individual en oposición a otro grupo de unos tres especialistas que realizan tratamientos en paralelo. La mayor diferencia, por lo que hemos podido conocer hasta ahora, es que los segundos realizan donación de óvulos de una paciente a otra mientras los primeros

${ }^{5}$ Hasta ahora se han realizado tres entrevistas. Una de las mujeres entrevistadas (a quien dimos el seudónimo de Sandra) es madre de dos hijos gestados por reproducción in vitro. Se realizó los tratamientos a fines de la década de los años noventa y el segundo hijo fue producto de la inseminación de un óvulo congelado, fruto del tratamiento para lograr su primer embarazo. Además tiene un tercer hijo que se gestó sin que mediara tratamiento alguno. Otra tuvo en febrero de 2012 mellizos por inseminación artificial (Laura) y la tercera (Andrea) hace diez años que se realiza tratamiento de reproducción asistida, incluyendo dos inseminaciones artificiales en una de las cuales ofició de donante de óvulos de otra mujer la cual hoy es madre gracias a esta inseminación.

${ }^{6}$ Se trata de una reflexión primaria ya que entendemos que aún faltan realizar algunas entrevistas. De todos modos los y las médicos/as que trabajan en reproducción asistida en nuestro país no suman más de 8 , nosotros hemos entrevistado a tres. 
rechazan este procedimiento prefiriendo que, en caso de necesitar óvulos, se recurra a una donante anónima pero que no esté en tratamiento,

[...] creemos que no puede compartirse toda una cosa que puede ser una expectativa del óvulo que cuando a vos te toca, la mujer se sacó seis y te tocan tres para vos y tres para vos, puede ser que te salga menos el procedimiento, pero las chances de tener son pocas [...] Y entonces creo que la donante tiene que ser donante. Si no se presta a cosas que no son muy claras.

Y más adelante en la misma entrevista explica,

Hay un programa, que se llama Programa Ovodona, que lo llevan dos médicos de acá y del grupo nuestro, que no tiene nada que ver con los que llevamos las pacientes adelante. Entonces lo que hacemos es, no tener contacto directo con la ovodonante, yo no la conozco. La paciente mia, que viene a ser la receptora de esa donación, la manejo yo en la conducción, me pongo de acuerdo con él qué fecha tiene tu paciente, y la hago menstruar el mismo día que la otra.

En la entrevista realizada a la doctora que trabaja en la misma línea que el anterior, ella también refirió a la ovodonación,

Se buscan mujeres que estén en condiciones de ser donantes. Se busca que tenga un perfil altruista. No digo que sea garantido el perfil altruista, porque hay dinero en el medio. Es mucho lo que tienen que hacer, yo no lo haría, por más buena que sea. "Esperá un momento, si tengo que perder de ir a trabajar, me van a pinchar, por lo menos dame algo a cambio". Entonces por lo general es el perfil de una mujer altruista, que ya tiene hijos y que dice yo estoy perdiendo óvulos todos los meses si a alguien le pueden servir yo se lo doy "no, pero mirá que te tenés que", "ah, no", "pero mirá que te regalan mil dólares", "ah, bueno, está bien, porque le tengo que hacer la fiesta de cumpleaños al nene", y le hace con los mil dólares el cumpleaños al nene, que antes no lo podía hacer. Esto desde el punto de vista de cómo conseguir la donante.

Retomando la constatación de Stolcke (2006) sobre el papel fundamental que juega la disponibilidad de ovocitos en el desarrollo de estas tecnologías pero también en la posibilidad de concepción, las diversas maneras en que se maneja la donación en ausencia de un marco legal crean diversas formas de vulnerabilidad e inequidades.

Antes de continuar quisiéramos volver al texto del proyecto de ley de 2003 para señalar algunos aspectos centrales del mismo, entre ellos la prohibición de la ovodonación. 


\begin{tabular}{|r|l|}
\hline $\begin{array}{r}\text { Carpeta Comisión de Salud, } \mathbf{n}^{\circ} \mathbf{3 1 8 1 .} \\
\text { Año 2003 }\end{array}$ & \\
\hline Destino de los embriones & Permite crio-preservación \\
\hline Determinación de la filiación & Social \\
\hline Donación de óvulos & No autoriza \\
\hline Elegibilidad & Parejas heterosexuales estables \\
\hline Gestación de substitución & No autoriza \\
\hline $\begin{array}{r}\text { Número máximo de embriones que se } \\
\text { permite transferir }\end{array}$ & 3 \\
\hline Reducción embrionaria & No menciona \\
\hline
\end{tabular}

Fuente: elaboración propia en base a los ejes conceptuales manejados por Diniz (2003)

La finalidad de este proyecto, según su propio texto, era favorecer la solución de los problemas reproductivos derivados de la esterilidad o de la infertilidad humana de la pareja (artículo $6^{\circ}$ ). El sujeto al cual se buscaba atender era una pareja heterosexual estable que haya recibido el diagnóstico de infertilidad o esterilidad. Porque aunque en el artículo $6^{\circ}$ se señala que "[...] en casos no ocasionales de impotencia, infertilidad masculina extrema o azoospermia del esposo o concubino se podrá recurrir a las técnicas aceptadas para la utilización de material reproductivo de terceros" lo que se contempla es la pareja como unidad a la cual se le brinda ese material reproductivo. En este caso se procura que el dador tenga la máxima similitud fenotípica e inmunológica y las mayores posibilidades de compatibilidad con la mujer receptora. La identidad del donante es en ese caso anónima y solo se podrá revelar en circunstancias que comporten un comprobado peligro para la salud sicofísica grave del hijo o cuando el hijo cumpla la mayoría de edad (18 años)

Las fuentes en las cuales se fundamenta el referido proyecto provienen de países como Estados Unidos (1980), Australia (1982), Francia (1984), el comité Ad Hoc de Expertos en Bioética de la Comunidad Europea (1989), y la legislación española de 1988. Es esta última, según se expresa en la exposición de motivos, más precisamente la Ley n ${ }^{\circ} 35$ (España, 1988), la base del proyecto uruguayo en el entendido de que existen criterios de afinidad cultural.

Si bien, como antes mencionamos, este proyecto no fue aprobado, su texto da cuenta de una serie de supuestos culturales de base. En tal sentido proponemos tomar para nuestro análisis el campo discursivo en el cual convergen el texto del proyecto de ley, los discursos de los/as médicos/as entrevistados/as en los cuales dan cuenta de sus prácticas - reconociendo la distancia que existe entre lo que se hace y lo que se dice que se hace-, y los de las mujeres entrevistadas. Volvamos ahora a los ovocitos y su circulación. 


\section{Ovocitos: el cuello de botella de la reproducción asistida}

No todas las mujeres con "buenos" óvulos están dispuestas a ser donantes ni todas las mujeres que no poseen buena cantidad y calidad de ovocitos aceptan ser receptoras de este material genético proveniente de otra mujer. Sin embargo, las que se encuentran en el segundo caso no tienen otro camino que aceptar la ovodonación, ya que las otras opciones serían no tener descendencia o adoptar. Entre las primeras, en cambio, están aquellas que donan para obtener una retribución económica, como señalaba la médica entrevistada, aquellas que ni se plantean la donación, esto es, la mayoría de las mujeres en edad fértil, y aquellas otras que tienen una buena producción de óvulos pero no los recursos económicos para costearse un tratamiento de reproducción asistida. ¿Cuáles son los discursos que se erigen para que una mujer intercambie sus óvulos para costear parte de su tratamiento de reproducción asistida? Y paralelamente, ¿cuáles es la barrera cultural, de sentido, que impide o dificulta la decisión de donar óvulos?

Cuando en el segundo tratamiento de fertilización in vitro el médico le sugirió a Andrea la posibilidad de donar óvulos para otra pareja en tratamiento en la cual la mujer carece "de buenos óvulos" y de esa manera poder afrontar parte de los gastos de su tratamiento, lo hizo, según la entrevistada, en los siguientes términos: "yo sé que te suena raro, pero es como dar en adopción un hijo. [Yo escuchaba] porque, claro, es persona a la que vos le donás los folículos, esos folículos van a ser fecundados con el semen del marido, ella lo va a tener en la panza, pero la información genética de ella no va a haber. Va a ser un hijo tuyo con ese marido".

Sandra, otra de las entrevistadas reflexiona asimismo sobre la donación de óvulos, aunque ella nunca estuvo en la situación — que ella define como "límite"—para hacerse ese planteo,

ENTREVISTADORA: ¿Alguna vez te propusieron?

SANDRA: No, porque nunca tuve que, que, no llegué a ese límite. Pero siempre prensaba si los tuviera que donar, y no los hubiera donado.

ENTREVISTADORA: ¿Qué es lo que no te convence?

SANDRA: No me convence ir por la calle un día y encontrar un nene igual a mi, que es hijo mio... o sea, realmente me choca. Como tampoco tendría un hijo con un óvulo de otra mujer o con esperma de otro hombre. Son esas cosas que nunca me las planteé, que no me gustan, para eso adopto... digamos. Yo qué sé.

Podríamos preguntarnos con Mujica (2009), ¿es la vida del ser viviente el equivalente a la vida de la persona?, o en este caso más aún, ¿es el material genético el equivalente a la vida de la persona?

Con el advenimiento de las TRA uno de los temas éticos que se vuelve central, fundamentalmente en las discusiones de la década de los años ochenta, es el estatuto del embrión. El estatuto del embrión pone sobre el tapete temas como la posibilidad de intervenir el feto de manera intrauterina incluso a pesar de la negación de la ges- 
tante a ser intervenida (Le Breton, 1995) ${ }^{7}$, la cantidad de embriones a implantar y el desecho de aquellos "sobrantes", la posibilidad o no de utilizar embriones para fines de investigación y el tiempo para el mantenimiento de embriones congelados. En su análisis sobre el estatuto del embrión, Salem (1997) se pregunta sobre el tiempo socialmente tolerado para la manutención de un embrión congelado y señala que estas y otras respuestas dependerán de la visión que se tenga acerca del embrión, visiones que la autora divide en tres posiciones:

1. Quienes alegan que el embrión es desde su concepción un ser moral con derecho a la vida y por tanto se oponen a todo tipo de manipulación.

2. Quienes sustentan que no existe diferencia alguna entre el embrión y otros tejidos humanos y que por tanto no hay que poner límites a la manipulación e investigación.

3. Una posición intermedia que defiende que, aunque el embrión no tiene el mismo estatuto que un niño ni un adulto, sí tiene el carácter de persona humana potencial y en tal sentido debe ser merecedor de consideraciones especiales.

Recalca también la autora que la manera de hablar respecto al embrión dice de nuestra manera de concebirlo. Los relatos de las entrevistadas nos permitirían afirmar, a modo de hipótesis exploratoria, que existe en el plano cultural cierta homologación entre embrión y ser humano.

Como dice Habermas (2002) hay una percepción cultural de la vida humana prenatal, y la medicina y su saber biologicista, no escapan a esto.

Andrea finalmente decidió donar ovocitos para una paciente en tratamiento y así costear parte de su tratamiento in vitro. Su decisión fue justificada y auto-justificada en el deseo que ella y su pareja tienen de poder tener un hijo biológico. Apelar a la maternidad y a determinado tipo de conformación familiar - heterosexual, formalmente constituida y con descendencia biológica-, situaciones de gran valor cultural en nuestras sociedades occidentales modernas, permite a Andrea "aliviar" la carga de llevar adelante una decisión que no está culturalmente aceptada y que ella, siendo parte de esa cultura, tampoco acepta. La negociación moral en este caso apela al discurso de la maternidad como el valor de mayor cuantía, pero es este mismo discurso el que genera otras desigualdades para otras personas en relación a la reproducción asistida.

${ }^{7}$ Le Breton menciona que en Estados Unidos existe la posibilidad de que los médicos apelen a la justicia para obligar a una mujer embarazada a operarse si se considera que la intervención es necesaria para proteger al feto e incluso hacerle una cesárea prematura para salvar al niño. "La maternidad está, entonces, disociada y la madre es percibida como el continente del feto. El aislamiento de la gestación del ser de la madre permite la redefinición jurídica del vínculo madre-feto" (Le Breton, 1995:238) Si la madre negó la intervención —y los médicos no pudieron realizarla - en el futuro el niño puede judiciar a su madre porque le negó una vida mejor. 


\section{El importunismo de quienes mantienen vínculos "naturalmente infértiles".}

¿Por qué muchas mujeres deciden someter sus cuerpos a múltiples intervenciones y a un proceso de medicalización sumamente exigente para lograr una descendencia "biológicamente vinculada" (Diniz y Gómez, 2006) ¿ ¿Por qué no optar por otros caminos como la adopción o la no descendencia? ¿La maternidad es realmente un deseo propio o un deseo ajeno y apropiado — en el sentido más amplio de la palabra: de apropiárselo y apropiado socialmente-?

Como destaca Imaz (2010) la reproducción se concibe en nuestro universo cultural como una evidencia y lo que desde las ciencias sociales suele abordarse son aquellas situaciones en las cuales se pone en juego esta situación natural: las parejas que eligen no tener descendencia, las tasas de natalidad por debajo del nivel de reemplazo, los abortos. El deseo de maternidad aparece como tan incuestionable que nadie tiene respuestas concretas acerca de las razones para materializar este deseo difuso, no objetivado y vinculado a ciertos valores e imaginario social, en un deseo de hijo, esto es, "[...] un deseo concreto y focalizado en una hija o hijo individualizado y propio" (ibid: 118) El pasaje por un tratamiento de reproducción asistida, ¿permite objetivar el deseo de maternidad? Y en este proceso de desnaturalización, ¿permite visualizar las injusticias por las cuales quienes pasan por este proceso transitan y más aún en relación a aquellos que desearían poder transitar tal camino no pueden hacerlo por diversos motivos?

Como han destacado muchos teóricos (Badinter, 1991; Barrán, 1993; Jelin, 1998; Nari, 2004) la idea de que todas las mujeres desean ser madres y que la maternidad es el acontecimiento más importante en la vida de una mujer e indispensable para su realización personal, no es más que una construcción ideológica que se fue naturalizando como parte del entramado cultural occidental, de la mano de la fuerte medicalización y concomitante control del cuerpo femenino a lo largo del siglo XX.

Estas presiones socioculturales para la realización de la maternidad en las mujeres, y el hecho de que la mayoría de las mujeres por estar socializadas también en esa urdimbre cultural incorporan estas ideas sin cuestionamientos para elaborar sus propios deseos y biografías, es un elemento central en relación a la desigualdad de género en el campo de la reproducción y, para este caso en particular, el de la reproducción asistida.

${ }^{8}$ Este concepto de Diniz y Gómez Costa (2006) permite mirar la finalidad de aplicación de las TRA de manera diferente a como lo hace la Organización Mundial de la Salud, para la cual la infertilidad es una enfermedad. Para Diniz y Gómez Costa lo que las TRA realizan no es curar una enfermedad, incluso en la mayoría de los casos se logra la concepción sin conocer la causa de la infertilidad o sin revertirla, sino que el objetivo está en producir un hijo biológicamente vinculado. Asimismo es este tipo de relación, el vínculo biológico, lo que define el tipo de maternidad que se desea; como destaca Badinter (1991), las concepciones acerca de la maternidad dependen de cómo se establezcan las relaciones en una tríada compuesta por la madre, el padre y el bebé. 
Así la imposición de la maternidad como horizonte de realización de las mujeres conduce — entre otros factores - , a inscribir esa ausencia de deseo de ser madre ${ }^{9}$ en un universo de sentidos que se contrapone a lo culturalmente esperable.

Por otra parte resulta de interés indagar cómo la búsqueda activa $a^{10}$ de la concepción produce cambios en el compromiso de los varones hacia el logro de este objetivo.

Tamanini (2009) nos brinda, en un estudio detallado sobre la aplicación de las nuevas tecnologías de reproducción asistida en Brasil y las vivencias de quienes transitan estos procesos para lograr la reproducción, algunos indicadores de las continuidades y rupturas que podrían estar ocurriendo en relación al compromiso de varones y mujeres en tales trayectorias: [...] se percebe como está entrando o homem a ser medicalizado, já que é uma escolha que ele debe fazer e sustentar ao longo do processo, ainda que a medicalización continue prioritariamente ocurrendo sobre o corpo feminino [...]" (Tamanini, 2009: 115). La autora señala que en el campo de las TRA existen un conjunto de valores y representaciones en relación a la paternidad que están cambiando: las antiguas resistencias al tratamiento se están flexibilizando, la asociación entre infertilidad e impotencia cuestionando y, el papel social del padre también está adquiriendo nuevas significaciones.

El rol cada vez más participativo de los varones en la crianza de los hijos —en el proceso de paternaje ${ }^{11}$ - , parece entonces estar habilitando cambios en su disposición a someter sus cuerpos a estas tecnologías y paralelamente, de la medicina reproductiva de tenerlos como sujetos destinatarios del proceso de medicalización.

Laura, quien tuvo mellizos hace poco tiempo gracias a un tratamiento de inseminación artificial, señala acerca del compromiso de su marido,
LAURA: [...] adelgazó cuarenta kilos.
ENTREVISTADORA: ¿Cuarenta kilos?!
LAURA: Sí, le habían dicho que la gordura era mala paraaa, que podía influir...
ENTREVISTADORA: ¿Él hizo un tratamiento para eso?
LAURA: Si [...] fue a un especialista, pero él tampoco tenía nada como grave. Pero al haber adelgazado. La doctora nos dijo que en la última inseminación yo tenía todos los valores perfectos, él estaba más flaco, estábamos mejor supuestamente y no sé si fue por eso o fue porque tenía que ser...

\footnotetext{
${ }^{9}$ No nos referimos a una ausencia de deseo en términos abstractos, sino en relación a la situación concreta de embarazo y el significado que tiene ese evento para la persona en un momento y contexto cultural, social, personal, vincular... determinado.

${ }^{10}$ Proponemos este concepto para colocar la concepción en tanto problema y no consecuencia directa del ejercicio de la sexualidad.

${ }^{11}$ La participación en estos procesos sin embargo no implica la transformación en relación a otras esferas que hacen el dominio doméstico de los varones sobre las mujeres. Como señala Siquiera (1999 en Tamanini (2009)), los varones no dejan su lugar de autoridad en la familia sino que mantienen un doble patrón de moral sexual. Ello más allá que, desde la sociedad y quienes elaboran las políticas sociales, la responsabilidad sobre el cuidado de los hijos siga recayendo en las mujeres.
} 
Más allá de tales cambios, las mujeres siguen teniendo un lugar central en relación a la reproducción y lo que ésta conlleva. En el caso de la reproducción asistida este sitial se materializa en la mayor medicalización de sus cuerpos y en la relevancia del útero como lugar de desarrollo, nutrición y cuidado de la futura criatura y por tanto como órgano cuya capacidad será la que en definitiva determinará el éxito de un tratamiento de reproducción asistida. Por otra parte, el Proyecto de Ley uruguayo, como se ilustra en el cuadro precedente, impide la gestación subrogada reafirmando así la relevancia del útero. De todas maneras en la entrevista con uno de los médicos especialistas en el área pudimos saber que muchas parejas uruguayas gastan cuantiosas sumas de dinero por una gestación subrogada en Estados Unidos, por lo cual aunque la misma no está ajena a nuestro horizonte de posibilidad cultural, sí lo está para muchos según sus posibilidades económicas.

\begin{abstract}
ENTREVISTADORA: ¿Cuáles son los lugares?
MEDICO: En Nueva York y en California, en California hay una organización que se llama "Madres sustitutivas". Tienen toda una preparación, deeee, la lista de receptoras quiénes son, cuáles características físicas tienen, hacen el contacto con ellas, les dicen cómo tienen que ir, cuándo tienen que ir, qué fecha tiene que tener la madre para ello, son mujeres jóvenes, generalmente. Sale muy caro, sale entre 60 y 70 mil dólares todo el procedimiento, y también tenés que contar con que si falla tenés que hacerlo de nuevo y esteee, porque no siempre es exitoso, pero bueno (Médico entrevistado).
\end{abstract}

\title{
5. Los inoportunos
}

Así como estos discursos dejan en un rango de importancia menor otras demandas, como son las de adopción por parte de parejas gays y lesbianas, en la dimensión doctrinaria que hace a las relaciones de género $\left(\right.$ Scott, 1996) ${ }^{12}$, la normativa relativa a la reproducción asistida en Uruguay coloca al margen de la posibilidad de acceso a las TRA a personas con determinada adscripción o que ocupan ciertos lugares sociales. Nos referimos a las mujeres solteras, a los menores de edad, a quienes no tengan una pareja heterosexual con estabilidad en el tiempo y aquellos que tienen una relación definida como homosexual. De este modo generan una injusticia social mediante el mecanismo de la exclusión: construyendo una línea simbólica a la vez que material que separa a aquellos que "pertenecen" (en este caso, que deberían ser contemplados en sus demandas) y aquellos que "no pertenecen" (o no deberían ser contemplados): "[...] inqualities are outcomes of action. They do not derive just from sources, whatever the argumentation. Inequalities are produced, reproduced, reduced, and dismantled by social interaction" (Therborn, 2006:11)

${ }^{12}$ El género debe ser comprendido según Scott como una categoría analítica con dos dimensiones interrelacionadas. Una dimensión relativa a las relaciones sociales basadas en las diferencias que distinguen los sexos; en esta dimensión se ubican: los símbolos culturalmente disponibles que invocan representaciones múltiples de lo masculino y femenino; los conceptos normativos para la significación de los símbolos (doctrinas); las nociones políticas, organizaciones e institucionales y la identidad subjetiva. La segunda dimensión refiere al género como forma primaria de relaciones significantes de poder; como un campo de articulación del mismo. 
Como señalábamos antes, el deseo de ser madre o padre se construye y a esa construcción o deseo se lo viabiliza o no de acuerdo a si cumple con ciertos requerimientos sociales y culturales que el deseo debe obedecer. Existe una primera desigualdad que atañe a lo que pueden desear algunas personas y no otras, o por lo menos los deseos que pueden obtener respuesta desde la dimensión socio cultural normativa siempre atravesadas por las consideraciones de género: aquellas parejas que no se ajustan a los preceptos de la heteronormatividad no deberían desear una descendencia. Los límites culturales del deseo en el ámbito de la reproducción deja fuera del acceso a las TRA a todos estos sujetos para quienes la reproducción - retomando la idea de Imaz (2010) de la evidencia de la misma - , no se presenta en la "naturaleza de sus relaciones sexuales" de manera natural. La injusticia que sufren estos sujetos es básicamente un asunto de reconocimiento, consecuencia del heterosexismo predominante en nuestra matriz socio-cultural que construye normas que privilegian la heterosexualidad de mano de la homofobia o la devaluación cultural de la homosexualidad (Fraser, 1998)

En el proyecto de ley uruguayo sobre Reproducción Humana Asistida, la obligatoriedad de que quienes se sometan a un proceso de este tipo conformen una pareja heterosexual estable está explícitamente mencionada: Los procedimientos de reproducción sólo podrán ser solicitados por parejas heterosexuales estables. En cada caso deberá firmarse la solicitud correspondiente. Desde ese momento los solicitantes son los responsables de todas las formas biológicas, derivadas de la utilización de estas técnicas durante todas las etapas, hasta el nacimiento (Artículo $6^{\circ}$ ).

Como señala Lagarde (1990) la sexualidad está en la base del poder: tener una u otra definición genérica implica para los seres humanos ocupar un lugar en el mundo. Independientemente de la voluntad, la adscripción genérica ubica: es una forma de integración en la jerarquía social; significa también tener y ejercer poderes sobre otros, o no tener siquiera poder sobre la propia existencia.

Se define así una primera desigualdad entre seres humanos por su lugar en un entramado socio cultural atravesado por los preceptos de género. Se les niega un reconocimiento, lo cual representa una injusticia no solo por no poder obtener lo que otras personas sí pueden obtener, sino - y fundamentalmente- porque se les está diciendo que no pueden obtenerlo porque no se les considera personas dignas de ello; "[...] tal comportamiento es dañino porque impide a estas personas tener una comprensión positiva de sí mismas - comprensión que se adquiere en la intersubjetividad - " (Axel Honneth, 1992:188-189; en Fraser, 1998:22).

Para paliar esta forma de injusticia se precisan soluciones transformativas, que apunten a una transformación de la estructura cultural valorativa subyacente (Fraser, 1998), un cambio en el entramado cultural que nos permita apreciar la capacidad de personas implicadas en arreglos familiares no tradicionales para ejercer el maternazgo o el paternazgo ${ }^{13}$, lo cual supone como movimiento en paralelo, una relativización de la relevancia para la vida de las personas de la consecución de

${ }^{13}$ Refiere a la responsabilidad emocional en relación a los/as hijos/as; la crianza y el cuidado de los/as mismos/as. 
ciertos mandatos culturales como la maternidad para que desde una distancia cultural crítica puedan deconstruir el deseo de producir una descendencia biológicamente vinculadas para eventualmente reconstruirlo de una manera autónoma.

También existe una situación de injusticia cuando se somete a la mujer destinataria de las TRA a un conjunto de requisitos que la coloca en una situación de menoscabo de sus derechos con respecto a las demás mujeres que en esta sociedad sostienen un embarazo sin recurrir a estas técnicas, mujeres a las cuales no se les solicita probar su idoneidad como madres (Fassler, 1999). En el caso de la adopción también se somete a la familia a una serie de requisitos. Sin embargo, en uno y otro caso, existe una diferencia que debemos considerar: cuando se trata de adopción lo que se están otorgando son los derechos y deberes concernientes a la patria potestad sobre otra persona, persona ya existente a la cual el Estado debe asegurar bienestar. En la otra situación la persona no existe.

Moviéndonos a un costado de lo que sucede en Uruguay y viendo también lo que con respecto al acceso a las TRA ocurre en otras partes del mundo, en un artículo publicado en la revista Human Reproduction, se menciona la solicitud de la Sociedad Europea de Reproducción Asistida a que se rechazara a las mujeres bebedoras, obesas y fumadoras para los tratamientos de reproducción asistida (Invitrotv.com). Este pedido de rechazo se basa en algunos argumentos como que el alcohol se relaciona con tasas bajas de embarazo y malformaciones, la obesidad con riesgo de diabetes durante la gestación y el tabaco con mayores posibilidades de sufrir un aborto espontáneo. Por ello, también se señala, la reproducción asistida debería realizarse con la condición de un cambio de estilo de vida. Por un lado se les está pidiendo a estas mujeres que dejen ciertos hábitos si es que quieren tener posibilidades de acceso a las TRA ${ }^{14}$, y por otro lado, la apelación a razones al parecer científicamente fundadas, opaca el hecho de que también la ciencia es una construcción cultural que reproduce los preceptos sociales y culturales dominantes.

Como señala Jaris Mujica, con respecto al instrumento que simboliza los saberes científicos indubitables, el microscopio, este símbolo de la ciencia moderna no es sin embargo independiente del ojo que mira y de los saberes que éste arrastra consigo: "[...] El microscopio no es un instrumento que implique por sí mismo 'objetividad', pues amplía el tamaño de los objetos, pero no necesariamente modifica la perspectiva de quien observa" (Mujica, 2009: 55) ${ }^{15}$.

Resulta interesante analizar estos mismos preceptos o normativas con respecto al caso mencionado anteriormente del varón que adelgazó cuarenta kilos para lograr la concepción. ¿Era realmente necesario o se trata de parte del control sobre cuerpos, tanto femeninos como masculinos, que busca ejercer el poder médico? ¿Se puede definir qué es lo necesario para lograr lo concepción o todo ello hace parte

${ }^{14}$ Es verdad que también se les pide a las mujeres que se embarazan sin intermediación de estas tecnologías ciertos cambios en conductas que puedan resultar perjudiciales para la gestación, pero el realizar o no estos cambios dependerá en último término de que las mujeres lo asuman o no, y el no asumirlo no significa que necesariamente se produzcan consecuencias negativas.

${ }^{15}$ Cursivas en el original. 
del universo de sentido, relaciones y poderes que la implementación de las TRA van construyendo? ¿Avanza este discurso hacia la igualdad de responsabilidad entre varones y mujeres en el terreno de la reproducción?

\section{La asistencia para la maternidad como contralor de conductas indeseables}

A su vez, como todos los órdenes socio culturales, el campo de la reproducción asistida no se desarrolla al margen de las desigualdades económicas, las cuales se interseccionan (Viveros, s/f) con otras de las múltiples desigualdades mencionadas.

¿Qué supone el concepto de "interseccionalidad"? Permite asumir que las desigualdades, en este caso de género y económicas, no son producto de la convergencia, fusión o adición de distintos criterios de discriminación (Dorlin, 2008; en Viveros, s/f) sino que permiten analizar la sobredeterminación de unos criterios sobre otros - en los ejemplos que estudia Viveros la sobredeterminación sexual de las categorías racistas que permiten afirmar, por ejemplo, que tanto las mujeres como los varones negros están natural y sexualmente siempre dispuestos-, así como la dinámica de articulación de las diversas desigualdades.

Si nos centramos en el acceso a las TRA, podemos aventurar que existe una sobredeterminación de la dimensión económica por sobre los mandatos de género: la maternidad es alentada para aquellas mujeres que se sitúan en los estratos socio económicos con mayores recursos mientras debe limitarse y se visualiza como un problema social entre aquellas poblaciones de menores recursos. Esta idea de que la nación se reproduzca bajo el signo del "bien nacer" fue la que guió las políticas de población de corte eugenista entre los años 1918-1939 en el Río de la Plata, como bien documenta Sapriza (2001) en su tesis de maestría, y que en los hechos se concretó en la despenalización del aborto en Uruguay por un breve lapso temporal, en el control de las epidemias, la regulación de la inmigración en América Latina, las leyes protectoras de la mujer como madre, la protección a la infancia y el control de enfermedades hereditarias a través de la planificación de los matrimonios.

El Estado actúa en lo referente a la prevención de embarazos entre la población de bajos recursos y se mantiene al margen en lo referente a brindar asistencia en materia de reproducción asistida para estas poblaciones. Uruguay, junto con Chile, se habían constituido en los dos únicos países en América Latina que brindaban asistencia en tecnologías de reproducción asistida en América Latina. Sin embargo, la clínica que funcionaba con tal fin en Uruguay debió cerrar sus puertas al poco tiempo de comenzar su actividad, a mediados de 2010. Además la clínica brindaba de forma gratuita el tratamiento pero no la medicación, que podía conseguirse a un precio más accesible que en las clínicas privadas pero no siempre accesible para la población atendida, y por otra parte luego del tratamiento no estaba previsto un soporte de contención económica y social para el caso de que se produzca, como es habitual para estos casos, un nacimiento múltiple.

Las técnicas más sencillas de reproducción asistida, como la estimulación ovárica, tienen en clínicas privadas en Uruguay un costo cercano a los setecientos dólares por cada vez que se realice el tratamiento, que puede alcanzar una periodicidad 
mensual hasta lograr la concepción; las más complejas rondan los cuatro mil dólares más dos mil dólares de medicación.

Si bien se calcula que aproximadamente un 15 por ciento de la población de nuestro país padece trastornos reproductivos no se cuenta con datos de cuántas de ellas desean acudir a TRA y cuántas realmente pueden enfrentar los costos que estos tratamientos suponen.

Las parejas de bajos recursos están además, también en esta materia, sujetas a un sistema de atención que las deja en una situación de injusticia permanente. Ya no se trata de que no puedan acceder en términos económicos a las TRA, sino que muchas veces no pueden siquiera acceder a los estudios que permitan evaluar su situación reproductiva. Como señalaba una conocida militante feminista con ocasión de la discusión del Proyecto de Ley de Reproducción Asistida,

[...] 134 parejas estériles han solicitado asistencia en el Centro Nacional de Estudios de Esterilidad y Fertilidad Humana. De ellas el 51,5 por ciento están casadas y el 48,5 por ciento en concubinato. En general, son familias de bajos recursos que no pueden costear los gastos de la atención en clínicas privadas, pero deben pagar el precio de la ausencia de recursos con la que trabajan las dependencias estatales y esperar, por ejemplo, un plazo promedio de tres meses para realizarse una laparoscopia (uno de los tantos análisis previos necesarios para el diagnóstico de las posibles razones de infertilidad) (Abracinskas, 1998, párrafo 9).

La sobredeterminación económica también se visualiza para el caso de las parejas homosexuales que quieren tener una descendencia biológicamente vinculada. Como advierten Diniz y Gómez Costa (2006), en Argentina los criterios de negociación moral en el campo de la sexualidad dentro de la esfera mercantil ${ }^{16}$, es decir, los criterios de elegibilidad para un tratamiento de fertilización asistida en el ámbito privado son más laxos que en la esfera pública, mostrando así la primacía de la economía sobre los derechos de las personas.

En Uruguay, según los testimonios de los médicos y las médicas entrevistados/ as, al no existir un marco legal vigente también se asiste a parejas homosexuales y a mujeres solas. Justamente es sobre la situación de las mujeres solas que reflexiona uno de los médicos entrevistados,

MEDICO: Normalmente las parejas que vienen acá son, mujeres solas, que vienen ENTREVISTADORA: ¿Que quieren tener hijos o quieren criopreservar?

MEDICO: Unas quieren preservar óvulos y otras quieren tener una inseminación donante.

ENTREVISTADORA: ¿Eso es posible?

MEDICO: Al principio el banco no daba muestras para mujeres solas. Yo lo convencí al, al médico Dr. *** que es el laboratorio del banco de esperma y el Dr. *** al principio estaba reticente a eso y le digo "mirá, es tan claro como que la mujer se hace el seguimiento folicular, y se va a Buenos Aires y se insemina, entonces no

${ }^{16}$ Según Diniz y Gómez Costa (2006), para el caso de Argentina, la restricción del acceso a las mujeres homosexuales o solas en la medicina pública es mayor que en las clínicas privadas. 
veo por qué estás negando la posibilidad de que además hoy en día estoy convencido de que la mujer tiene el derecho a tener un hijo. Porque esa mujer sola, hemos tenido trillizos, que se hicieron una fertilización, que hoy deben tener 16 ó 17 años, y el marido puso la muestra para la fertilización, puso la muestra y se fue. Entonces digo, la mujer los crió... una maestra era". Entonces digo la mujer creo que tiene el derecho de decidir su vida, como cualquier ser humano, y no lo quiere tener por acostarse con cualquiera, me parece que puede ser una buena chance.

Las palabras del entrevistado dan cuenta, por un lado, de la necesidad que se tenía de brindar una solución a una situación que de todas maneras iba a encontrar una respuesta del otro lado del Río de la Plata con la consecuencia de una pérdida de oportunidad económica para los médicos uruguayos. Pero brinda asimismo testimonio de ciertas transformaciones que se están operando en el terreno de la cultura: la posibilidad de que una mujer pueda concebir sin necesidad de un varón, trasladar al plano biológico lo que de hecho sucede en el ámbito social ya que muchas veces el progenitor no se responsabiliza por la concepción y el nacimiento de una criatura.

\section{Otras desigualdades: guetización del conocimiento y ponderación de los temas de agenda}

Cualquier ley que regule la reproducción humana asistida debería realizarse dentro de un marco globalizador en lo que se refiere a derechos sexuales y reproductivos. Por eso este proyecto es parcial, “...dado que no hay políticas sobre educación sexual ni sobre el control de la natalidad, estaríamos ante una política estrictamente localizada en materia demográfica, destinada a un porcentaje muy restringido de la población [...]" (Lilián Abracinskas, en Dos Santos, 1997)

Si no se visualiza la reproducción asistida en un marco de construcción de derechos reproductivos de manera integral, las consecuencias en cuanto al ejercicio de derechos entre mujeres que se asisten para lograr la reproducción y aquellas que pasan por un proceso de reproducción fuera de las TRA termina siendo la generación de diferentes capacidades de incidencia sobre el proceso reproductivo para cada una de las situaciones. Por ejemplo: en el proceso de reproducción asistida se contempla la intervención sobre el cigoto para detectar y eventualmente tratar enfermedades hereditarias; si existe alguna "anomalía" puede indicarse la no transferencia de este cigoto al útero para su implantación. En una mujer que queda embarazada sin recurrir a las TRA y que descubre, a partir de una ecografía o de un estudio del líquido amniótico, que su futuro bebé tiene alguna alteración cromosómica o directamente es inviable, no puede abortar dentro de un marco legal.

Es interesante pensar aquí sobre el concepto de "reproducción estratificada" (Rapp, 2001) en tanto el mismo alude a cómo las tecnologías biomédicas reproducen una organización jerárquica de la salud reproductiva, la fecundidad y las experiencias de nacimiento, patologizando algunas experiencias ${ }^{17}$. Y si bien el concebir

${ }^{17}$ En tal sentido resulta de interés la patologización que se realiza del dolor durante el parto a partir de la introducción de la analgesia para el trabajo de parto. 
mediante tratamiento de reproducción asistida genera una desigualdad en términos de poder actuar sobre el feto, desde una interpretación centrada en la dimensión de la cultura deberíamos interrogarnos acerca de si el conocimiento de determinada condición del feto y la posibilidad de abortarlo supone un mayor acercamiento al ejercicio de los derechos reproductivos. Por otra parte el margen de actuación sobre el feto que habilita esta posibilidad interroga el concepto de "feto supervalioso", concepto que los y las médicos/as utilizan para referirse al producto de una gestación que fue posible gracias a una fecundación in vitro y que justifica maniobras como la cesárea programada para minimizar cualquier riesgo. Al decir de uno de los médicos entrevistados:

[...] puede haber un parto normal. Pero lo que sí es minimizar los riesgos. Porque lo que se llama "feto supervalioso" no es porque es el feto de esa paciente, sino porque fue muy difícil conseguirlo. Entonces las pacientes que consiguen esto buscan tener todas las precauciones para que al feto no tengan que hacerle un fórceps y después una cesárea porque no salió por fórceps, entonces eso aumenta un poco el índice en las estériles de las cesáreas. Porque son planificadas el día que sale, la hora, neonatólogo. Todo.

El "feto supervalioso" aparece como el producto de un proceso en el cual todo sucedió dentro de lo esperado; un proceso que, en tanto altamente controlado, podría si no haber sido interrumpido.

Otras desigualdades que terminan en injusticias son relativas al acceso al conocimiento y formación en tales técnicas por parte de los profesionales de la salud. Como señalaba Moriana Hernández, activista feminista en el marco del proceso de discusión del Proyecto de Ley de Reproducción Asistida,

[...] consideramos necesario recomendar que la Facultad de Medicina amplíe sus estudios curriculares sobre la materia, de lo contrario, esto se transformaría en un monopolio encubierto porque solo aquellos profesionales que tienen la capacidad y los recursos económicos necesarios para adquirir estas técnicas en el exterior, podrían instalar una clínica [...] (Hernández, en Dos Santos, 1997)

Si bien el acceso a la educación universitaria no es equitativo para toda la población, incluso en un país como Uruguay que cuenta con una Universidad de la República de carácter gratuito y con sistemas de apoyo a quienes tienen mayores dificultades de acceso, la guetización de ciertos saberes para un conjunto de personas con determinadas posibilidades socio económicas no hace más que reproducir un status quo que favorece la reproducción de ciertos colectivos y pautas de dominación.

\section{Reflexiones para continuar la reflexión}

Hemos avanzado, de manera muy somera, algunas reflexiones sobre los cambios que en Uruguay se están procesando en relación a la aplicación de las TRA en un contexto complejo en tanto no existe un marco jurídico que regule el accionar de los técnicos y técnicas del área. 
La aproximación a tal contexto, a partir de la propuesta legislativa, el discurso de los y las especialistas y algunas mujeres que han recibido tratamientos, permiten avanzar algunas líneas de pensamiento que se intentarán seguir profundizando en comparación también con otras realidades en las cuales también se aplican estas tecnologías.

Algunos testimonios permiten pensar en un mayor compromiso de los varones en el proceso de reproducción y paralelamente la cada vez mayor aceptación de que la mujer tome por cuenta propia decisiones reproductivas. Sin embargo, deberíamos cuestionarnos acerca de si estos cambios se producen en el marco de un avance de la equidad en las relaciones de género o si obedecen a la búsqueda de parte de la medicina de una cada vez mayor ampliación del control sobre los cuerpos y su capacidad reproductiva, además de las ganancias derivadas de la aplicación de las TRA.

Por otra parte se erigen nuevas desigualdades que obedecen a viejos modelos. Las construcciones de género imperantes generan modos de exclusión específicos para ciertos colectivos que se sitúan en los márgenes de lo culturalmente valorado; las conductas sexuales son un elemento fundamental dentro de tal ordenamiento y quienes se sitúan en los márgenes de lo esperado en relación a estas conductas no son considerados/as para el acceso a las TRA.

Las sociedades occidentales modernas evalúan los actos sexuales según un sistema jerárquico de valor sexual. En la cima de la pirámide erótica están solamente los heterosexuales reproductores casados. Justo debajo están los heterosexuales monógamos no casados y agrupados en parejas, seguidos de la mayor parte de los demás heterosexuales. [...] Las parejas estables de lesbianas y gays están en el borde de la respetabilidad, pero los homosexuales y lesbianas promiscuos revolotean justo por encima de los grupos situados en el fondo mismo de la pirámide. Las castas sexuales más despreciadas incluyen normalmente a los transexuales, travestís [...] (Rubin, 1989:18)

Este ordenamiento jerárquico también es un modo de organizar la desigualdad (Therborn, 2006) y genera mecanismos de exclusión que no son iguales para todos los que se ubican en un lugar u otro de la jerarquía. Una pareja homosexual con recursos económicos puede eventualmente pagar un tratamiento de reproducción asistida; si no es en Uruguay, cruzando el Río de la Plata ${ }^{18}$. Es en tal sentido que la búsqueda del reconocimiento cultural-valorativo va de la mano de una redistribución económica (Fraser, 1998).

Por otra parte, si comparamos las situaciones de injusticia para cada una de las dimensiones, económica y de género, entre parejas de bajos recursos o solo para el caso de las parejas homosexuales podemos visualizar diferencias. En el primer caso se yergue una barrera material infranqueable o difícilmente transitable para el caso de que existiera una legislación que atienda a las situaciones mencionadas. En el segundo se trata de una barrera simbólica que como tal es permeable a ciertas

${ }^{18}$ En conversaciones informales para el trabajo de tesis he sabido de parejas homosexuales que se asisten en Buenos Aires. 
concepciones y en este sentido se somete a una lógica simbólica atravesada por la omnipresencia del mercado.

Pero además, las parejas homosexuales que acuden a clínicas para realizarse un tratamiento de fertilización asistida no buscan soluciones transformativas, como diría Fraser (1998), sino paliar una situación personal que en la dimensión de la injusticia económica lo que hace es continuar reproduciéndola.

Como señala la Viveros (s/f), la interseccionalidad es inherente a toda relación de dominación e impide o debilita las tentativas de resistencia. En este caso podríamos señalar que la demanda por un acceso equitativo en términos económicos a las TRA se ve constreñido por la lógica individualista del sistema capitalista que indica que quien puede pagar los costos para llevar adelante algunos de los tratamientos no se ocupe de que quienes no pueden pagar estos costos tenga un acceso pleno a los mismos; o en los casos de la conformación de un grupo demandante del acceso a estas técnicas no busquen ampliar sus demandas hacia otros colectivos cuya situación de inequidad responde a razones que ellos no entienden como propias.

Así la existencia y aplicación de las TRA en Uruguay replantea nuestras formas de concebir la reproducción, sus condiciones y productos, de una manera que no se presenta unívoca en cuanto a la ampliación de los márgenes de libertad para los sujetos.

\section{Referencias bibliográficas}

\section{ABRACINSKAS, Lilián}

1998 "Hacia dónde va la reproducción Humana". Cotidiano Mujer, 26. Disponible en: http://www.cotidianomujer.org.uy/1998/parasalu26.htm. (accedido el 5 de septiembre de 2009)

AMADOR JIMENEZ, Mónica

2010 "Biopolíticas y biotecnologías: reflexiones sobre maternidad subrogada en India", en: CS No. 6, julio-diciembre. Cali, Colombia, 193-217.

BADINTER, Elizabeth

1991 ¿Existe el instinto maternal? Barcelona: Paidós.

BARRÁn, José Pedro

1993 Medicina y Sociedad en el Uruguay del Novecientos. Montevideo: Banda Oriental.

BUTLER, Judith

2003 “O parentesco é sempre tido como heterossexual?”, en: Cadernos Pagú, № 21. Brasil: Universidad de Campinas.

DINIZ, Debora; GÓMEZ COSTA, Rosely

2006 "Infertilidad e infecundidad: acceso a nuevas tecnologías reproductivas", en: Cáceres, Carlos F. et al. (Orgs.). Sexualidad, estigma y derechos humanos: desafios para el acceso a la salud en América Latina. Lima: FASPA/UPCH, 55-67.

DOS SANTOS, Lupe

1997 "Un mundo Feliz". Cotidiano Mujer, 25. Disponible en: http://www.cotidianomujer.org.uy/1997/feliz25.htm. (accedido el 5 de noviembre de 2010) 
FASSLER, Clara

1999 "Legislación sobre técnicas de reproducción Humana Asistida". Cotidiano Mujer, 30. Disponible en: http:// www.cotidianomujer.org.uy/1999/clara.htm (accedido el 5 de noviembre de 2010)

FRASER, Nancy

1998 Iustitia Interrupta. Reflexiones críticas desde la posición "postsocialista”. Bogotá: Siglo de Hombre Editores.

HABERMAS, Jurgen

2002 El futuro de la naturaleza humana. Hacia una eugenesia liberal. Barcelona: Paidós.

HARAWAY, Donna

1991 "A Cyborg Manifesto: Science, Technology, and Socialist-Feminism in the Late Twentieth Century", en: Simians, Cyborgs and Women: The Reinvention of Nature. New York: Routledge, 149-181.

IMAZ, Elixabete

2010 Convertirse en madre. Etnografía del tiempo de gestación. Madrid: Cátedra.

$\mathrm{S} / \mathrm{A}$

Invitrotv.com (2010) Piden rechazar el tratamiento de reproducción asistida a mujeres obesas y a las que beban en exceso. Disponible en: http://www.invitrotv.com/ noticia/1329/piden-rechazar-el-tratamiento-de-reproduccion-asistida-a-mujeresobesas-y-a-las-que-beban (accedido el 27 de diciembre de 2010)

JELIN, Elizabeth

1998 Pan y afectos: la transformación de las familias. Buenos Aires: Fondo de Cultura Económica.

LAGARDE, Marcela

1990 Los cautiverios de las mujeres: madresposas, monjas, putas, presas y loca. México D.F: Coordinación General de Estudios de Postgrado de la UNAM.

LE BRETON, David

1995 Antropología del cuerpo y la modernidad. Buenos Aires: Nueva Visión.

MINES, Diana

2002 "Que se vayan todas. Los partidos políticos ante el desafío de la maternidad lesbiana". Cotidiano Mujer, 38. Disponible en: http://www.cotidianomujer.org. uy/2002/38_p38.htm. (accedido el 5 de noviembre de 2010)

MUJICA, Jaris

2009 Microscopio. De la bioética a la biopolítica. Lima: Centro de defensa y promoción de los derechos sexuales y reproductivos (Promsex).

NARI, Marcela

2004 Políticas de maternidad y maternalismo político. Buenos Aires: Biblos. 
RAPP, Rayna

2001 "Gender, Body, Biomedicine: How Some Feminist Concerns Dragged Reproduction to the Center of Social Theory", en: Medical Anthropology Quarterly, New Series, Vol. 15, No. 4, Special Issue: The Contributions of Medical Anthropology to Anthropology and Beyond (Dec., 2001).

REYGADAS, Luis

2008 La apropiación. Destejiendo las redes de la desigualdad. Barcelona y México: Antropos.

RUBIN, Gayle

1998 "Reflexionando sobre el sexo: notas para una teoría radical de la sexualidad", en: Vance, Carole S. (Comp.) Placer y peligro. Explorando la sexualidad femenina. Madrid: Revolución, 113-190.

SAPRIZA, Graciela

2001 La "utopía eugenista”. Raza, sexo y género en las políticas de población en el Uruguay (1920-1945). Tesis de Maestría. Facultad de Humanidades y Ciencias de la Educación. Universidad de la República.

SCAVONE, Lucila

2007 Novas Tecnologias Conceptivas: teorias e políticas feministasen: Verônica Ferreira Maria Betania Ávila Ana Paula Portella (orgs) Feminismo e Novas Tecnologias Reprodutivas. Recife: SOS Corpo, Instituto Feminista para a Democracia, 13-23.

SALEM, Tania

1997 "As novas tecnologias reprodutivas: o estatuto do embrião e a noção de pessoa", en: Revista Mana, vol. 3, abril, $\mathrm{n}^{\circ} 1$. Rio de Janeiro.

SCOTT, Joan W.

1996 "El género: Una categoría útil para el análisis histórico", en: Lamas, Marta (Comp.) El género: la construcción cultural de la diferencia sexual. México-PUEG, 265-302.

SCHWARZ, Patricia K.N.

2009 "La maternidad y el maternazgo en el cuerpo de mujeres jóvenes argentinas". Ponencia presentada en la VI Reunión de Antropología del Mercosur, Buenos Aires. Publicada en formato CD.

STOLCKE, Verena

2006 "La mujer es puro cuento. La cultura del género. Desarrollo Económico, Vol. 45, $N^{\circ}$ 108. Buenos Aires: Instituto de Desarrollo Económico y Social, 523-546.

THERBORN, Goran

2006 "Meaning, Mechanisms, Patterns, and Forces: An Introduction", en: Therborn, Goran (ed.) Inequalities of the world. Londres: Verso, 1-58.

VIVEROS VIGOYA, Mara

s/f "La sexualización de la raza y la racionalización de la sexualidad en el contexto latinoamericano actual". Disponible en: http://ucaldas.edu.co/docs/seminario_familia/ Ponencia_MARA_VIVEROS.pdf (accedido el 3 de enero de 2011) 